\title{
Development of Degree-of-Priority Based Control Strategy for Emergency Vehicle Preemption Operation
}

\author{
Jiawen Wang, Wanjing Ma, and Xiaoguang Yang \\ Key Laboratory of Road and Traffic Engineering of the Ministry of Education, Tongji University, 4800 Cao'an Road, \\ Shanghai 201804, China \\ Correspondence should be addressed to Wanjing Ma; mawanjing@tongji.edu.cn
}

Received 22 August 2013; Accepted 5 November 2013

Academic Editor: Huimin Niu

Copyright (C) 2013 Jiawen Wang et al. This is an open access article distributed under the Creative Commons Attribution License, which permits unrestricted use, distribution, and reproduction in any medium, provided the original work is properly cited.

This paper proposes a degree-of-priority based control strategy for emergency vehicle preemption operation to decrease the impacts of emergency vehicles on normal traffic. The proposed model features its effectiveness to the following three aspects: (1) a multilayer fuzzy model was established to determine the degree-of-priority based on emergency vehicle preemption demand intensity and preemption influence intensity; (2) for emergency vehicles with proper classification, a travel time estimation model for emergency traffic was formulated, an optimal emergency route determines model based on the level of priority of emergency events, and the emergency vehicle travel time was developed to minimize evacuation time as well as minimize the adverse impacts of preemption on normal traffic; and (3) a conditional traffic signals priority control method at each intersection of the evacuation route was built, so that traffic queue at each intersection can be cleared before the arrival of emergency vehicles. A simulation model based on field data was developed, and the performance of the proposed strategy was compared with the conventional local detection based method under the microscopic simulation model. The results validated the efficiency of the proposed strategy in terms of minimizing the delay of emergency vehicles and reducing adverse impacts on normal traffic.

\section{Introduction}

Providing safe and fast driving environment for emergency vehicles to reduce travel time and delay is a critical issue in traffic evacuation. Under effective preemption, ones can reach their destinations at the earliest possible time which is one of most critical factors in saving lives and reducing property loss. At the same time, reducing the adverse impacts of emergency vehicles on normal traffic, so that they can cause the least disturbance to network traffic flow, is the key to avoid the grid-lock caused by emergency accidents $[1,2]$. While substantial progress has been made in the areas of vehicle detection and communication technologies to increase the efficiency of emergency vehicles, current stateof-the-art in signal preemption in China has not reached the point where signal clearance strategy, considering the adverse impact or normal traffic, can be automatically generated and implemented in real time.

To date, most preemption systems developed operate on a single-intersection basis and require local detection of an emergency vehicle to activate a signal preemption sequence at each intersection [3, 4]. Existing signal preemption methods can be classified into several categories such as optical, infrared light, acoustic, special types of loop detection, and GPS-based systems $[5,6]$. The optical systems that are developed in the 1960's use a strobe-lamp on the vehicle and an optical sensor per approach to an intersection requiring a clear line-of-sight path between the vehicle and the intersection [7]. The sound-based systems use the directional microphones installed at an intersection to detect the siren of vehicles approaching a given intersection; therefore, no special equipment is required for the emergency vehicles [8]. In a GPS-based system being operated in Taicang, China, both an emergency vehicle and an intersection are equipped with a GPS receiver and a radio transceiver for two-way communication. In recent studies a route-based dynamic strategy was developed for efficient preemption of traffic signals for emergency vehicles in real time by Kwon et al. [9] And Louisell and Collura [10] proposed a simple algorithm to estimate emergency vehicle travel time savings based on 


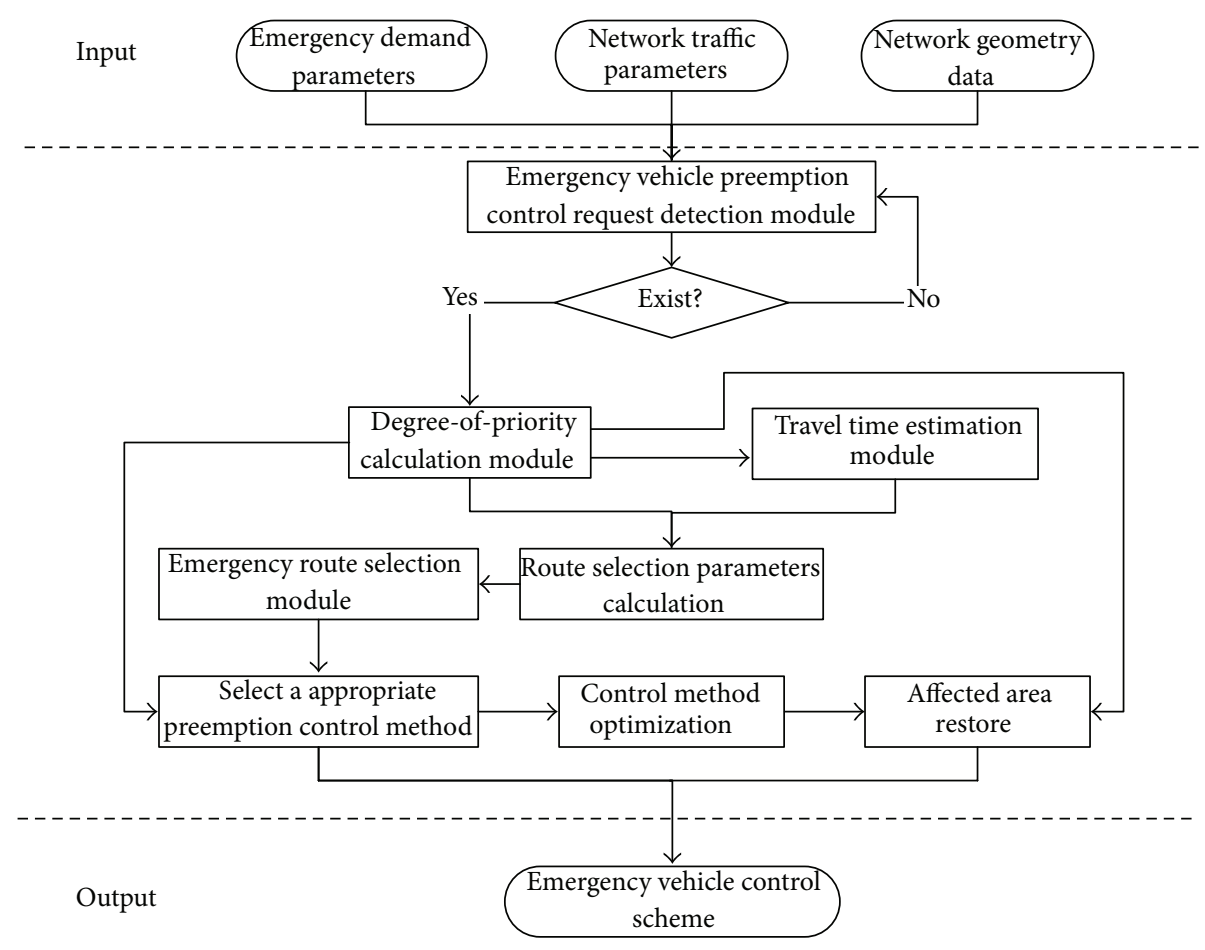

FIGURE 1: Structure of degree-of-priority based signal preemption strategies.

a field operational. Mussa and Selekwa [11] reported on the development of a transition procedure based on the quadratic optimization method that aimed at reducing disutility measures to motorists during the transition period. Haghani et al. [12] concentrated on developing an optimization model for developing flexible dispatching strategies that take the advantage of available real-time travel time information. In recent years, Yun et al. $[13,14]$ have optimized the exit phase controls for emergency vehicle preemption and compared the emergency vehicle preemption methods with a hardware-inthe-loop simulation. He et al. [15] presented a heuristic algorithm for traffic signal control with simultaneous multiple priority requests at isolated intersections in the context of vehicle-to-infrastructure communications being available on priority vehicles, and the method could reduce average bus delay in congested conditions by about $50 \%$. Savolainen et al. [16] developed a dynamically activated emergency vehicle alert system to provide an additional visual cue to motorists of an impending emergency vehicle's approach. Route selection is one of the fundamental problems in emergency logistics management [17-20]. In order to send the commodities as quickly as possible, the path which costs least should be selected. A vast amount of the literature [21-25] has been produced up until now.

As indicated above, while there has been substantial progress in developing local preemption technologies, and much research has focused on the impact of transit priority [26-28], the impact of EV preemption has not been well studied; a few research studies on decreasing the adverse impacts of preemption on normal traffic have been found in the literature. Qin and Khan [29] report two new control strategies for emergency vehicle signal preemption which reduce the response time and minimize the impact of $\mathrm{EV}$ operation on general traffic. Developing degree-of-priority based control strategy that can provide an efficient and safe traveling environment for emergency vehicles with minimum disruption on network traffic is of critical importance in managing urban traffic.

Based on the above achievements, this paper presents a dynamic preemption approach that combines a degree of control priority classification procedure and a route-based preemption method to provide the most appropriate route and control strategy for an emergency vehicle under a given network, traffic conditions, and emergency issue conditions. The proposed method was evaluated in part of the roads network in Beijing, China, using a microscopic network simulation model, and its effectiveness was compared with the existing local-detection based method.

This paper is organized as follows: the first part is the introduction of emergency vehicle preemption methods. The second part is the model of degree of priority classification and travel time prediction. The third part is development of emergency vehicle preemption strategy and performance evaluation. Last part is conclusion.

\section{Degree-of-Priority Based Signal Preemption}

Figure 1 shows the structure of the degree-of-priority based signal preemption strategy developed in this study. Input data include emergency demand parameters, network traffic parameters, and network data. Emergency demand parameters are obtained when emergency issue happens. 


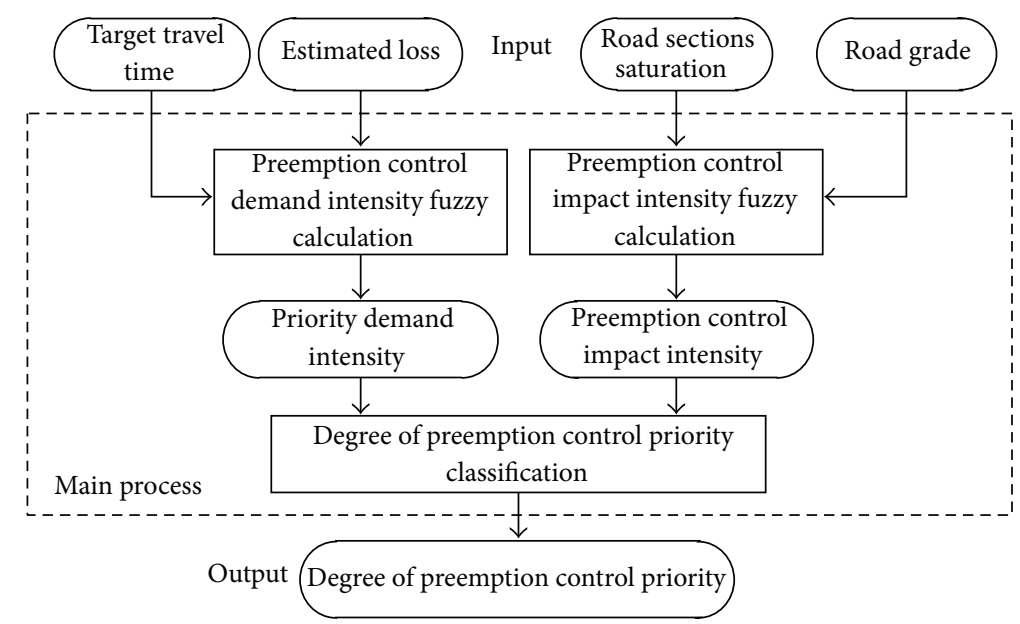

FIGURE 2: Emergency vehicle preemption signal control priority classification process.

Network traffic parameters are continuously collected by field detectors. Network geometry data are static data provided by government. Control strategy for emergency vehicle preemption will be started as soon as emergency issue happens, and the first step is classifying degree-of-priority of emergency vehicles in each road section, which corresponds to intensity needs of emergency vehicle preemption. Then travel time of emergency flow in each road section is predicted based on the input data and degree of priority. Next, two-objective optimization model is established to calculate route selection parameters and select the optimal route for emergency flow. In this research, the well-known Dijkstra algorithm [30] is adopted to find the optimal route that has the minimum route selection parameter for a given origin-destination pair. At last, signal control method is developed to optimize the emergency vehicle preemption plan, and affected area restore control plan is proposed.

\section{Degree of Priority Classification Model}

Degree of priority is an important parameter of emergency vehicle in this research, which corresponds to the important level and degree of influence of emergency vehicle in each road section. Because both importance level of emergency vehicle and degree of influence to normal network traffic flow are difficult to measure accurately, degree of priority could be calculated by the genetic algorithm or fuzzy algorithm. In this study, researchers developed a three-step, multilayer fuzzy algorithm to calculate the degree of priority in three steps.

To calculate preemption demand intensity, the seriousness and urgency of the emergency issue should be estimated based on desired travel time of emergency vehicle and estimated loss of the emergency issue. In the fuzzy reasoning of preemption demand intensity calculation, fuzzy inputs are the estimated loss parameter $P_{l k}$ of emergency issue $k$ and the urgency parameter $U_{k}$. Fuzzy output is preemption demand intensity $I_{\mathrm{pd}}$. For the convenience of computing, in this research the ranges of estimated loss parameter $P_{l k}$ and the urgency parameter $U_{k}$ are defined as 0 to 1 (see Figure 2).
The estimated loss parameter $P_{l k}$ can be calculated as follows:

$$
P_{l k}=\frac{\log \left(L_{k}+n L_{k}^{(P)}\right)}{10} .
$$

In (1), $P_{l k}$ is the estimated loss parameter of emergency issue $k . L_{k}$ is the estimated economic loss of emergency issue $k$ with $10,000 \mathrm{CNY}$ as its unit. $L_{k}^{(P)}$ is the conversion coefficient of casualties. $n$ is the estimated number of casualties. According to this formula, emergency events anticipated to have a higher estimated loss would result in a higher intensity of response.

The urgency parameter $U_{k}$ can be calculated as follows:

$$
U_{k}=1-\frac{\sum_{n=1}^{j}\left(L_{k n} / v_{m n}\right)}{T_{t}} .
$$

In (2), $U_{k}$ is the urgency parameter of emergency issue $k$. $T_{t}$ is the desired travel time of emergency vehicle appointed by decision makers. $L_{k n}$ is the length of road section $n$ in the shortest route $j . v_{m n}$ is the top speed of the emergency vehicle in road section $n$. From the formula we can find with the decrease of the urgency parameter $U_{k}$, the emergency vehicle is more urgent. It makes these two parameters fuzzy by membership function, as shown in Figures 3(a) and 3(b), to calculate the preemption demand intensity.

Fuzzy rules of preemption demand intensity classification are shown in Table 1(a). In Table $1,\{\mathrm{VH}, \mathrm{H}, \mathrm{M}, \mathrm{L}, \mathrm{VL}\}$ is short for $\{$ Very High, High, Medium, Low, Very Low\}.

The membership function of preemption demand intensity is triangular, and the range is $[0,1]$. Then, the exact value function of preemption demand intensity is obtained through the center-of-gravity defuzzification.

To consider the impact of emergency vehicle preemption on normal network traffic flow, preemption impact intensity is proposed to reflect the degree of this impact. Fuzzy inputs of preemption impact intensity fuzzy calculation are city road grade $v_{d j}$ of road section $j$ and saturation $x_{j}$ of road section $j$. The city road grade is divided into expressway, arterial road, 


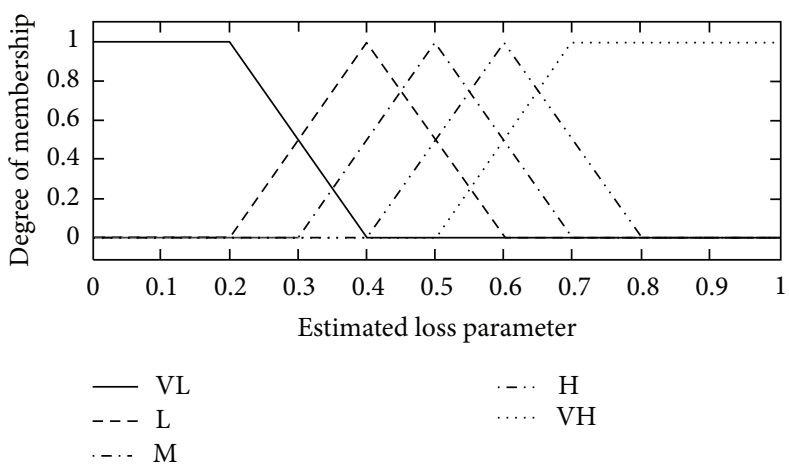

(a) Membership function of estimated loss parameter $P_{l k}$

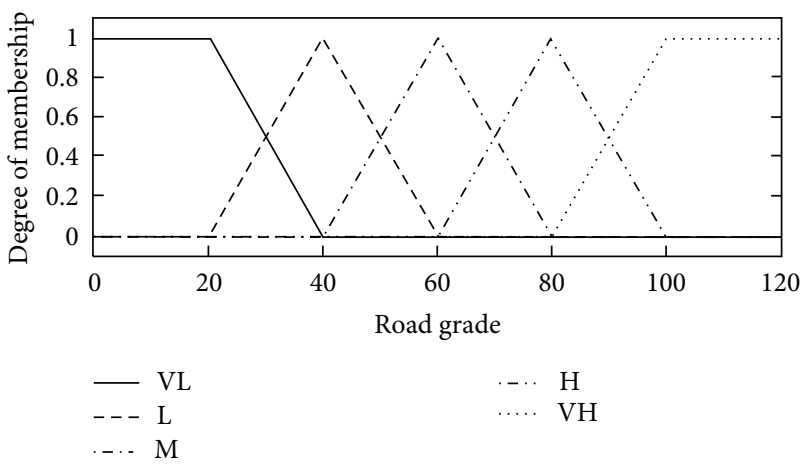

(c) Membership function of urgency parameter $U_{k}$

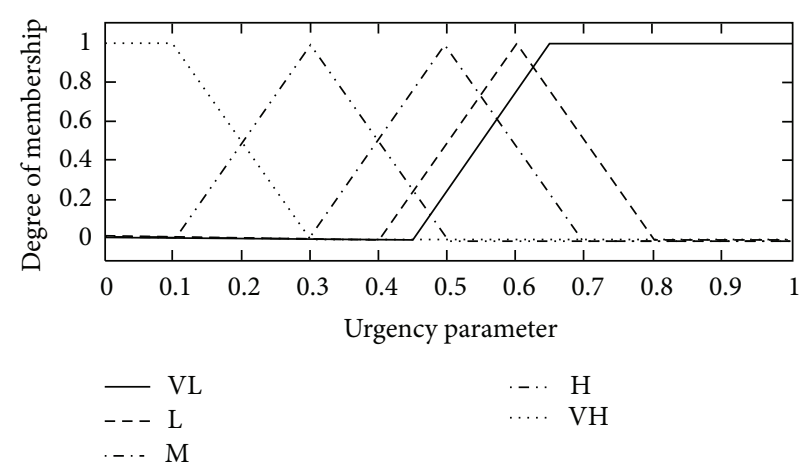

(b) Membership function of road grade $v_{d j}$

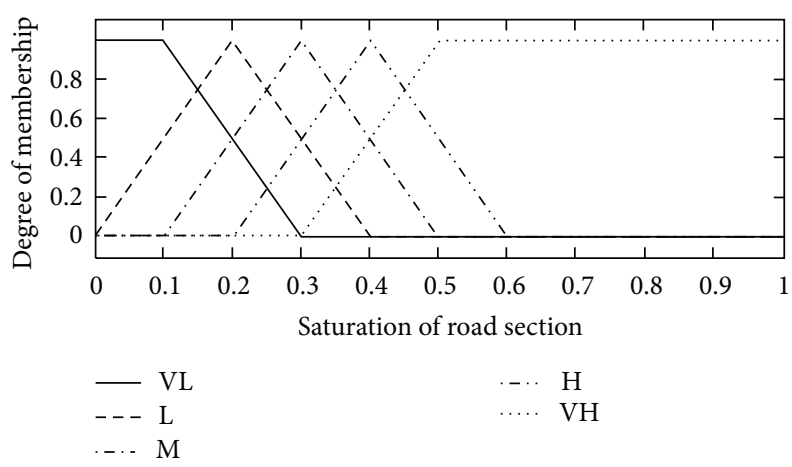

(d) Membership function of saturation $x_{j}$

FIGURE 3: Membership function in degree of priority classification model.

secondary trunk road, and branch road, which are indicated by value of $v_{d j}$. $\{80,60,40,30\}$ indicates $\{$ Expressway, Arterial Road, Secondary Trunk Road, Branch Road\}, respectively. Saturation of road section is calculated by volumes and capacity of the road section. It makes these two parameters fuzzy by membership function that is shown in Figures 3(c) and $3(\mathrm{~d})$ to calculate preemption impact intensity. Fuzzy rules of preemption impact intensity calculation are shown in Table 1(b).

The membership function of preemption impact intensity is triangular, and the range is $[0,1]$. Then, the exact value function of preemption impact intensity is obtained through the center-of-gravity defuzzification.

To calculate degree of priority of emergency vehicle on each road section, fuzzy rules of emergency vehicle preemption priority classification are used. The fuzzy rule is show in Table 1(c).

In Table 1(c), when preemption impact intensity is very high, the degree of priority is classified as a low value to avoid the impact on normal network traffic flow. And when preemption impact intensity is very low, normal network traffic flow volume is low. So the road impedance to emergency vehicle is low enough to classify degree of priority as a low value. Therefore, when Preemption Impact Intensity is medium, a high value is given according to these rules.

The membership function of emergency vehicle degree of priority is triangular, and the range is $[0,1]$. Then, the exact value function of emergency vehicle preemption priority is obtained through the center-of-gravity defuzzification. The emergency vehicle degree of priority $D_{p}$ is an important input in selecting control method.

\section{Travel Time Prediction Model for Emergency Vehicle}

Road section clearance time, $\Delta t$, is an important parameter that affects total travel time and delay of emergency vehicles. It stands for the time difference between the moment $t_{e}$ when the sections are changed into emergency state and the moment $t_{\text {in }}$ when the emergency vehicles enter the sections. The road section clearance time of different control methods is not the same, and it is related to emergency vehicle degree of priority of the road section. The higher degree of priority, the longer road section clearance time. In the urban road system, the difference of traffic characteristics vehicles will become discrete. The traffic flow setup from the upper intersection will become discrete, and the length of platoon will become long before the platoon arrives at the lower intersection. This phenomenon is called platoon dispersion. Previous studies have demonstrated that the speeds of vehicles follow Gaussian distributions.

In the multilane sections, lane-clear speed varies directly with mean time-headway. Lane-clear speed has the dimensions of $\mathrm{pcu} / \mathrm{km} \cdot \mathrm{s}$.

The lane-change behavior is very fast then the distance of platoon moving in the lane-change period is short, 
TABLE 1: Fuzzy rules in degree of priority classification model.

(a) Fuzzy rules of preemption demand intensity calculation

\begin{tabular}{lccccc}
\hline$U_{k}$ & & \multicolumn{3}{c}{$I_{\mathrm{pd}}$} & \\
& $P_{l k}: \mathrm{VH}$ & $\mathrm{H}$ & $\mathrm{M}$ & $\mathrm{L}$ & $\mathrm{VL}$ \\
\hline $\mathrm{VH}$ & $\mathrm{VH}$ & $\mathrm{VH}$ & $\mathrm{H}$ & $\mathrm{H}$ & $\mathrm{M}$ \\
$\mathrm{H}$ & $\mathrm{VH}$ & $\mathrm{H}$ & $\mathrm{H}$ & $\mathrm{M}$ & $\mathrm{L}$ \\
$\mathrm{M}$ & $\mathrm{H}$ & $\mathrm{H}$ & $\mathrm{M}$ & $\mathrm{L}$ & $\mathrm{VL}$ \\
$\mathrm{L}$ & $\mathrm{H}$ & $\mathrm{M}$ & $\mathrm{L}$ & $\mathrm{VL}$ & $\mathrm{VL}$ \\
$\mathrm{VL}$ & $\mathrm{M}$ & $\mathrm{L}$ & $\mathrm{VL}$ & $\mathrm{VL}$ & $\mathrm{VL}$ \\
\hline
\end{tabular}

(b) Fuzzy rules of preemption impact intensity calculation

\begin{tabular}{lccccc}
\hline$x_{j}$ & $v_{d j}: \mathrm{VH}$ & $\mathrm{H}$ & $\mathrm{M}$ & $\mathrm{L}$ & $\mathrm{VL}$ \\
\hline $\mathrm{VH}$ & $\mathrm{VH}$ & $\mathrm{VH}$ & $\mathrm{H}$ & $\mathrm{M}$ & $\mathrm{M}$ \\
$\mathrm{H}$ & $\mathrm{VH}$ & $\mathrm{H}$ & $\mathrm{M}$ & $\mathrm{L}$ & $\mathrm{L}$ \\
$\mathrm{M}$ & $\mathrm{H}$ & $\mathrm{M}$ & $\mathrm{M}$ & $\mathrm{L}$ & $\mathrm{VL}$ \\
$\mathrm{L}$ & $\mathrm{M}$ & $\mathrm{L}$ & $\mathrm{L}$ & $\mathrm{VL}$ & $\mathrm{VL}$ \\
$\mathrm{VL}$ & $\mathrm{L}$ & $\mathrm{VL}$ & $\mathrm{VL}$ & $\mathrm{VL}$ & $\mathrm{VL}$ \\
\hline
\end{tabular}

(c) Fuzzy rules of emergency vehicle preemption priority classification

\begin{tabular}{lccccc}
\hline$I_{\mathrm{pd}}$ & & \multicolumn{5}{c}{$D_{p}$} \\
& $I_{\mathrm{pi}}: \mathrm{VH}$ & $\mathrm{H}$ & $\mathrm{M}$ & $\mathrm{L}$ & $\mathrm{VL}$ \\
\hline $\mathrm{VH}$ & $\mathrm{VH}$ & $\mathrm{VH}$ & $\mathrm{VH}$ & $\mathrm{VH}$ & $\mathrm{VH}$ \\
$\mathrm{H}$ & $\mathrm{H}$ & $\mathrm{H}$ & $\mathrm{VH}$ & $\mathrm{VH}$ & $\mathrm{H}$ \\
$\mathrm{M}$ & $\mathrm{M}$ & $\mathrm{H}$ & $\mathrm{VH}$ & $\mathrm{H}$ & $\mathrm{M}$ \\
$\mathrm{L}$ & $\mathrm{L}$ & $\mathrm{L}$ & $\mathrm{M}$ & $\mathrm{L}$ & $\mathrm{VL}$ \\
$\mathrm{VL}$ & $\mathrm{VL}$ & $\mathrm{VL}$ & $\mathrm{L}$ & $\mathrm{L}$ & $\mathrm{VL}$ \\
\hline
\end{tabular}

so the distance is negligible. Total quantity of vehicles on road section $k$ can be calculated as follows:

$$
n_{k}=\frac{V_{k} L_{k}}{N_{k} v_{k}}
$$

In (3), $n_{k}$ is total quantity of vehicles on road section $k$. Parameter $V_{k}$ is the current volume of section $k$. Parameter $L_{k}$ is length of section $k$. Parameter $N_{k}$ is the number of lanes in section $k$. Parameter $v_{k}$ is mean travel speed of normal vehicles in section $k$. And quantity of vehicles that leave the lane in unit time $n_{k}^{\prime}$ is calculated as follows:

$$
n_{k}^{\prime}=\beta \mu L_{k} \frac{N_{k}}{V_{k}}
$$

In (4), Parameter $\beta$ is lane clear influence coefficient that represents the influence of different lane-clear methods on lane clear speed. Its value is defined as 1 when the lane-clear method is changing the lane from inside lane to outside lane in the two-lane sections. The value is demarcated by following simulation experience. And parameter $\mu$ is the ratio of laneclear speed to mean time-headway. The value is demarcated by following simulation experience.
Then we can find quantity of vehicles on the emergency lane after normal vehicle avoiding emergency vehicle $n_{e k}$ is calculated as follows:

$$
n_{e k}=\max \left(n_{k}-t_{k} n_{k}^{\prime}, 0\right)=\max \left(\frac{V_{k} L_{k}}{N_{k} v_{k}}-t_{k} \beta \mu L_{k} \frac{N_{k}}{V_{k}}, 0\right) .
$$

In (5), $t_{k}$ is road section clearance time. It is a function of emergency vehicle degree of priority $D_{p k}$. The formula is shown in (4). This formula should be calibrated based on field data in application. Consider the following:

$$
t_{k}=f\left(D_{p k}\right)
$$

The applications and studies of road section travel function are the most popular methods to estimate the emergency vehicle travel time by free flow speed, traffic volume, or capacity of the section. This study proposed an emergency vehicle travel time estimation model for the emergency vehicle control method based on bureau of public roads function (BPR function) [31]. BPR function was proposed by FHWA in 1964 in the US [32]. It is the most widely used impedance function in the traffic-planning field. In this function, travel time is the nonlinear function using the ratio of traffic volume to capacity as the parameter.

The definition of the public roads function is defined as follows:

$$
T_{k}=T_{0}\left[1+a\left(\frac{V_{k}(t)}{C_{k}(t)}\right)^{b}\right] .
$$

In (7), $T_{k}(t)$ is emergency vehicle estimated travel time in road section $k$ at $t$. Parameter $T_{0}$ is the travel time when the volume of section $k$ is zero. Parameter $V_{k}(t)$ is the volume of section $k$ at $t$. Parameter $C_{k}(t)$ is the capacity of section $k$ at $t$. Parameters $a$ and $b$ are coefficients whose advised values are $a=0.15$ and $b=4$.

Based on above BPR function, this study built the emergency vehicle travel time estimation model for the sections that are applied to different kinds of control methods as follows:

$$
T_{k}=\frac{L_{k}}{v_{e}}\left[1+a\left(\frac{n_{e k} N_{k} V_{k}}{L_{k} C_{k}}\right)^{b}\right] .
$$

In (8), $n_{e k}$ can be calculated by (5), then, one can get the following:

$$
T_{k}=\frac{L_{k}}{v_{e}}\left[1+a\left(\frac{\max \left(V_{k}^{2} L_{k}-\Delta t_{k} \beta \mu N_{k}^{2} v_{k}, 0\right)}{V_{k} L_{k} C_{k}}\right)^{b}\right] .
$$

In the formulation, (8) and (9), $T_{k}$ is emergency vehicle estimated travel time in section $k$. Parameter $V_{k}$ is current volume of section $k$. Parameter $L_{k}$ is length of section $k$. Parameter $N_{k}$ is the number of lanes in section $k$. Parameter $v_{k}$ is mean travel speed of normal vehicles in section $k$. Parameter $v_{e}$ is mean travel speed of emergency vehicles. Parameter $\beta$ is lane clear influence coefficient. 
Parameter $\mu$ is the ratio of lane-clear speed to mean timeheadway. Parameter $\Delta t_{k}$ is road section clearance time of section $k$. Parameters $a$ and $b$ are coefficients demarcated by following simulation experience.

If road section clearance time $\Delta t_{k}$ takes zero, that is to say, the emergency vehicle control method is disabled, $T_{k}$ can be calculated by the following equation:

$$
T_{k}=\frac{L_{k}}{v_{e}}\left[1+a\left(\frac{V_{k}}{C_{k}}\right)^{b}\right] .
$$

\section{Development of Emergency Vehicle Preemption Strategies}

5.1. Route Selection Parameter Calculation and Optimal Route Selection. In order to select the optimal route for emergency vehicle, route selection parameter should be calculated. The route selection method should provide an efficient and safe traveling route for emergency vehicle with minimum disruption on network traffic. And the disruption on network traffic of emergency vehicle declines with declining degree of priority. Therefore, the model has two optimization objectives including reducing route travel time to minimum and controlling the sum of degree of priority in the route to minimum. In this study, route selection parameter $P_{k}$ is defined as follows:

$$
P_{k}=T_{k}+\eta D_{p k}
$$

In (9), $T_{k}$ is emergency vehicle estimated travel time in road section $k . D_{p k}$ is emergency vehicle degree of priority in road section $k$. Parameter $\eta$ is degree of priority adjusting coefficients which will be determined in specific case.

Once the location of an emergency issue and emergency facilities (police stations, aid stations, etc.) is determined, the route-selection algorithm determines the best route that has the minimum route selection parameter for a given origin/destination pair. In the proposed strategy, a network is represented as a set of links/nodes and the well-known shortest-path algorithm developed by Dijkstra [30] is adopted to find the optimal route. Dijkstra's algorithm has been proven to result in the shortest-path from a single source on a weighted directed graph, where all edge weights have nonnegative values [33]. In the proposed strategy, a given network is modeled as a set of directional links with nonnegative route selection parameter $P_{k}$, and Dijkstra's algorithm is applied to find the minimum $P_{k}$ route.

5.2. Control Method Selection and Optimization. As soon as the emergency route is determined, this study proposes a strategy to select the control method based on degree of priority. Experience-based control method choice suggestion is shown in Table 2.

When degree of priority of the emergency vehicle is within 0.0 to 0.4 , no control method will be implemented to minimize negative effects of emergency vehicle on normal network traffic flow. And if degree of priority is within 0.4 to 0.7 , green-wave signal control method will be implemented to reduce travel time of emergency vehicle. Further, when
TABLE 2: Emergency vehicle control method choice suggestion.

\begin{tabular}{lc}
\hline Degree of priority & Control method \\
\hline $0.0-0.4$ & None \\
$0.4-0.7$ & Green-wave signal preemption \\
$0.7-0.9$ & Lane clearance preemption \\
$0.9-1.0$ & Road clearance preemption \\
\hline
\end{tabular}

degree of priority is within 0.7 to 0.9 , the degree of priority is high enough to implement lane clearance control method additionally based on green-wave signal control method. At last, if degree of priority is within 0.9 to 1.0 , related road sections will be temporarily closed to reduce travel time of emergency vehicle.

Determining the right offset times to activate the green signal for the intersections along the emergency route is of critical importance in reducing travel time of emergency vehicles and minimizing negative effects of signal preemption on normal traffic flow. As soon as the road sections that implement green-wave signal control method are determined for a given emergency vehicle, the signal offset time of related intersections is adjusted to ensure unidirectional green wave preemption. We record the target offset between downstream intersection $C_{d k}$ and upstream intersection $C_{u k}$ of road section $k$ as $O_{k}$. Then offset $O_{k}$ can be calculated as follows:

$$
\mathrm{O}_{k}=T_{k} \bmod C_{d k}
$$

Record the original offset between downstream intersection $C_{d k}$ and upstream intersection $C_{u k}$ as $O_{k}^{\prime}$. So the offset adjustment recorded as $\Delta O_{k}$ is calculated as follows:

$$
\Delta O_{k}=O_{k}-O_{k}^{\prime}
$$

If $C_{d k}<\sum_{i=0}^{k} T_{i}$, offset should be adjusted per cycle; $\Delta O_{\text {adj } k}$ is calculated as follows:

$$
\Delta O_{\mathrm{adj} k}=\frac{\Delta O_{k}}{\left\lfloor\sum_{i=0}^{k} T_{i} / C_{d k}\right\rfloor} .
$$

Else, offset should be adjusted per cycle $\Delta O_{\text {adjk }}=0$.

When the emergency vehicle departs, change the cycle of each downstream intersection to $C_{d k}-\Delta O_{\text {adjk }}$ until the offset is equal to target offset.

Lane clearance control method is an important method to reduce travel time of emergency vehicle further. Emergency lane is enabled by traffic police or variable lane control system when the emergency vehicle reache last road section. Startup time of emergency lane is determined by road section travel time of emergency vehicle.

\section{Performance Evaluation}

Finally the proposed degree-of-priority based control strategy is evaluated in a part of road network in Xicheng district, Beijing, China. Figure 4 is the diagram of the road network.

This area adopted SCOOT signalized intersections control system. Network traffic parameters and network data are shown in Table 3. 


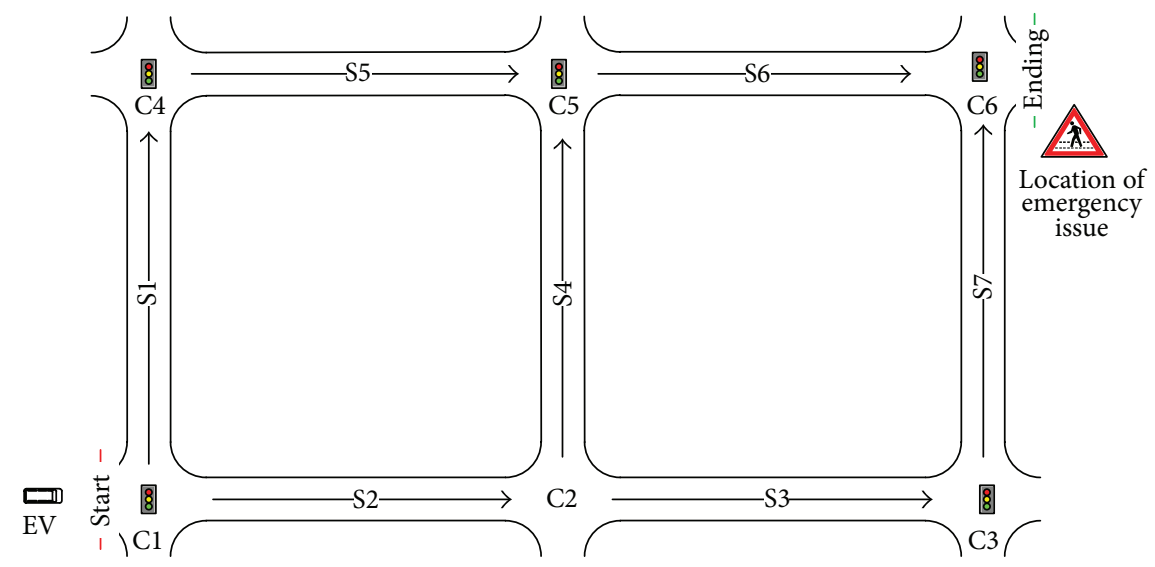

FIGURE 4: The simulation model of road network in beijing.

TABLE 3: Network parameters and data.

\begin{tabular}{|c|c|c|c|c|c|c|}
\hline \multirow{2}{*}{ Section name } & \multirow{2}{*}{ Section number } & \multirow{2}{*}{ Length (m) } & \multirow{2}{*}{ Road grade } & \multicolumn{2}{|c|}{ Traffic volume (PCU/h) 3/12 7:30-3/12 8:00 } & \multirow{2}{*}{ Capacity (PCU/h) } \\
\hline & & & & Direction of arrow & Reversed direction & \\
\hline Naoshikou Rd. & S1 & 517 & Arterial road & 1087 & 896 & 3200 \\
\hline West Xuanwumen Rd. & S2 & 445 & Arterial road & 684 & 768 & 2400 \\
\hline West Xuanwumen Rd. & S3 & 480 & Arterial road & 589 & 656 & 1600 \\
\hline Tonglinge Rd. & S4 & 485 & Branch road & 102 & 140 & 600 \\
\hline Xinwenhua Rd. & S5 & 442 & Secondary trunk road & 348 & 268 & 800 \\
\hline Xinwenhua Rd. & S6 & 477 & Secondary trunk road & 248 & 375 & 800 \\
\hline Xuanwumen Rd. & S7 & 463 & Arterial road & 1395 & 1674 & 3200 \\
\hline
\end{tabular}

In this case, fire occurs in a restaurant near intersection C6. Emergency vehicle that is composed of three fire trucks starts from fire station near intersection $\mathrm{C} 1$. To reduce loss, the desired travel time of emergency vehicle is set at $180 \mathrm{~s}$. The estimated loss is 2,000,000 CNY, and two of the injured are waiting to be rescued. A microsimulation model based on VISSIM is set up to calibrate some parameters in the strategies. The main processes of simulation are shown in Figure 5.

At a random time, the section in the simulator entered into emergency state which is shown in Figure 5(1). When the section was under emergency state, the vehicles on emergency lane changed to outside lane to clear a lane for the emergency vehicle. Then the emergency vehicle passed the intersection quickly under intersection signal control strategy. The former process is shown in Figures 5(2), 5(3), 5(4), and 5(5). According to the simulation, parameters of control strategy in this case are shown in Table 4.

Based on the degree-of-priority based control strategy and the data in Table 4, degree of priority and emergency vehicle travel time in each road section are calculated by Beijing Special Control System, which is developed by J+ traffic Tech Co. The results are shown in Table 5.

By using shortest-path algorithm developed by Dijkstra, the emergency routes based on different $\eta$ are shown in Figures 6(b) and 6(c).
In Figures 6(b) and 6(c) the red line indicates the road sections that adopt none of the control method, and the green line indicates the road sections that adopt green-wave signal control method. In this study, these two plans are evaluated in simulation software based on cellular automata developed by authors. The degree-of-priority based control strategy is compared with the local-detection based method in the software based on the same set of vehicle inputs. In the microsimulation traffic network, emergency vehicle detectors are set at $100 \mathrm{~m}$ from the intersection stop line. For a fair comparison, a common set of 15 different random seeds was used for the simulation of each preemption route, and their results were averaged. The simulation results are shown in Table 6.

As shown in Table 6, the emergency vehicle travel time under degree-of-priority based control strategy exhibits an indistinctive degraded performance. However, a clear pattern of the degraded performance in terms of average delay is exhibited under the degree-of-priority based control strategy. Along with degree of priority adjusting coefficient $\eta$ increase, thus the selected route is different, the average delay is declining in evidence. This indicates the efficiency of the proposed strategy by reducing unnecessary preemption in a given network, thus minimizing the delay because of preemption without decreasing much efficiency. 


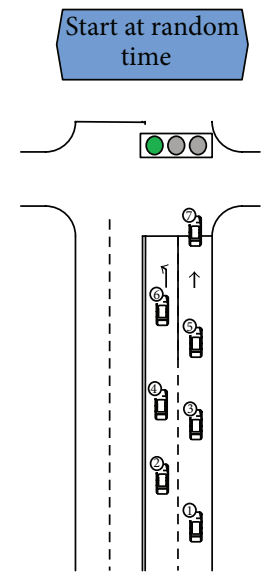

(1)

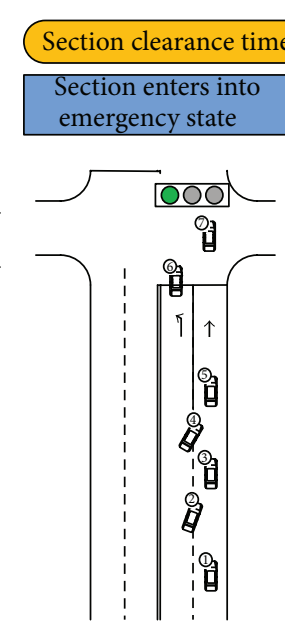

(2)

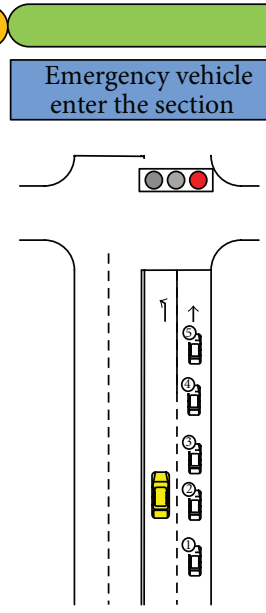

(3)
Travel time of emergency vehicle $t$

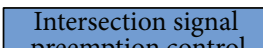

preemption control

Emergency vehicle

pass the intersection

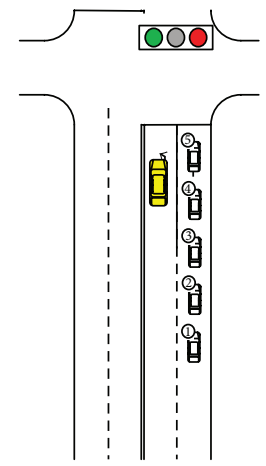

(4)

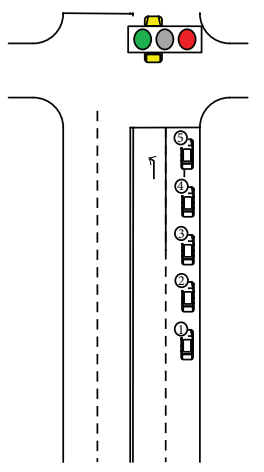

(5)

FIGURE 5: Main processes of simulation based on VISSIM.

TABLe 4: Parameters of control strategy in evaluation.

\begin{tabular}{lcc}
\hline Parameters & Symbol & Value \\
\hline Conversion coefficient of casualties & $L_{k}^{(P)}$ & 70 \\
Road section clearance time & $\Delta t_{k}$ & When $D_{p}<0.4 \Delta t_{k}=3$ \\
& & When $0.4<D_{p}<0.7 \Delta t_{k}=6$ \\
Lane clear influence coefficient & $\beta$ & Else $\Delta t_{k}=20$ \\
Ratio of lane-clear speed & $\mu$ & 1 \\
Coefficients of BPR function & $a$ & 135.7 \\
Coefficients of BPR function & $b$ & 1.96 \\
Degree-of-priority adjusting coefficients & $\eta$ & 1.59 \\
\hline
\end{tabular}

TABLE 5: Calculation results of degree-of-priority and emergency vehicle travel time.

\begin{tabular}{lcccccccccc}
\hline Section number & $x_{j}$ & $I_{\mathrm{pi}}$ & $I_{\mathrm{pd}}$ & $D_{p}$ & $\Delta t_{k}$ & $T_{k}$ & \multicolumn{2}{c}{$\eta$} & 50 & 100 \\
\hline S1 & 0.34 & 0.48 & 0.17 & 0.41 & 6.0 & 31.0 & 35.1 & 51.6 & 72.2 \\
S2 & 0.29 & 0.39 & 0.17 & 0.39 & 3.0 & 26.7 & 30.6 & 46.1 & 65.4 \\
S3 & 0.37 & 0.54 & 0.17 & 0.41 & 6.0 & 28.8 & 32.9 & 49.2 & 69.5 & 113.4 \\
S4 & 0.17 & 0.21 & 0.17 & 0.25 & 3.0 & 58.2 & 60.7 & 70.5 & 82.8 & 107.1 \\
S5 & 0.44 & 0.37 & 0.17 & 0.37 & 3.0 & 40.2 & 44.0 & 58.8 & 77.3 \\
S6 & 0.31 & 0.25 & 0.17 & 0.25 & 3.0 & 43.1 & 45.6 & 55.6 & 68.1 & 93.1 \\
S7 & 0.44 & 0.62 & 0.17 & 0.36 & 3.0 & 28.0 & 31.7 & 46.2 & 64.3 & 100.6 \\
\hline
\end{tabular}

TABLE 6: Simulation results from different preemption strategies.

\begin{tabular}{lccc}
\hline Routes & Item & Local-detection based method & Degree-of-priority based preemption \\
\hline \multirow{3}{*}{ Route 1 } & EV travel time (sec) & 103 & 106 \\
& Total vehicle hours & 11.3 & 10.8 \\
\hline \multirow{3}{*}{ Route 2 } & Average delay (sec) & 19.6 & 15.2 \\
& EV travel time (sec) & 133 & 142 \\
\hline \multirow{2}{*}{ Route 3 } & Total vehicle hours & 10.9 & 10.6 \\
& Average delay (sec) & 16.4 & 12.7 \\
& EV travel time (sec) & 159 & - \\
\hline
\end{tabular}




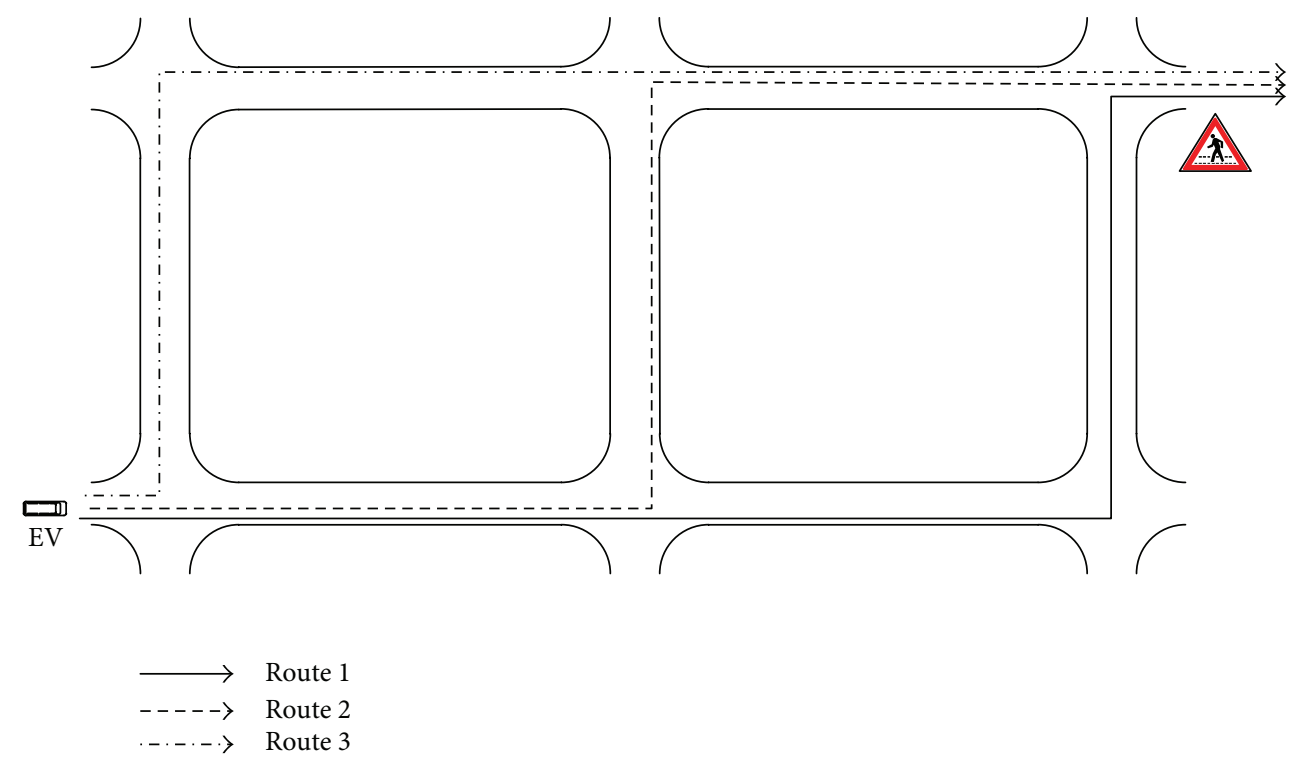

(a) Sample network

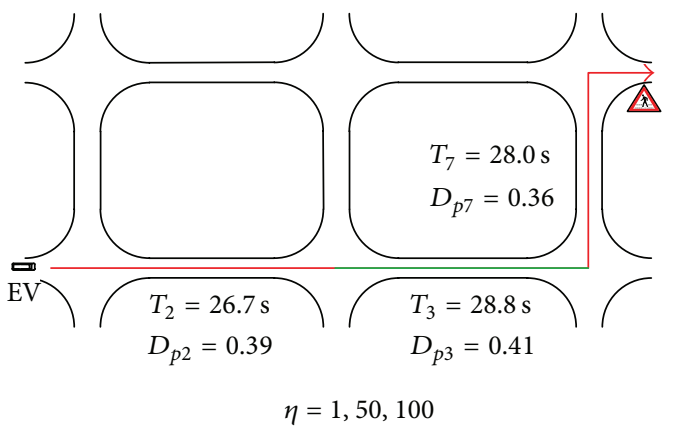

(b) Route 1

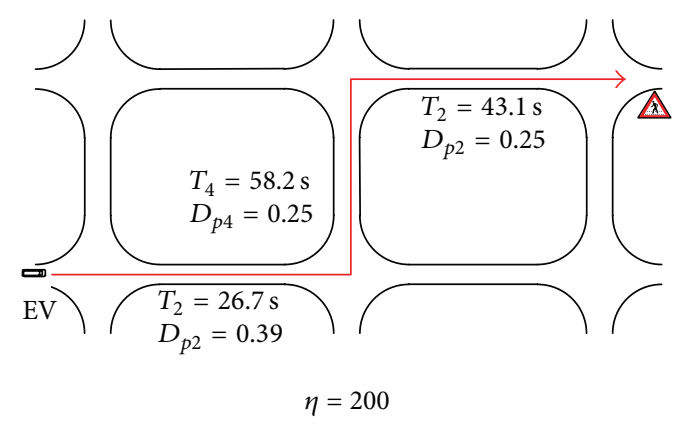

(c) Route 2

FIGURE 6: Sample network and selected emergency routes based on different $\eta$.

\section{Conclusions}

The primary objective of this paper is developing a degreeof-priority based control strategy for emergency vehicle operation to decrease the adverse impacts of emergency vehicles on normal traffic as well as avoid the grid-lock caused by emergency issues. A uniform optimization structure is designed which is consisted by a fuzzy logic based model that was proposed to determine the degree-of-priority of an emergency event. Then, an optimal emergency route selection model was built based on degree-of-priority and the estimated travel time of emergency vehicle. Four types of signal control methods were proposed to minimize the delay of emergency vehicles as well as adverse impacts on normal traffic. Therefore, the traffic signals at the intersections along the emergency route can be adjusted effectively in advance, and traffic queue at each intersection can be cleared for the approaching emergency vehicles. This strategy is evaluated in simulation software based on the data of a part of road network in Xicheng district, Beijing, China. The results indicate that the proposed approach is a viable addition to local-detection based method in urban area and can minimize the delay of emergency vehicles without much adverse effects on normal traffic. Future research includes the development of an efficient calibration method for the degree of priority with parameters which can be measured and the enhancement of the simulation model to evaluate the performance of the strategy in large road network.

\section{Conflict of Interests}

The authors declare that they have no conflict of interests regarding the publication of this paper.

\section{Acknowledgments}

This research was made possible through funding from the Ministry of Science and Technology of China under Grant no. 2011AA110305. The authors wish to acknowledge the vast and helpful assistance from Intelligent Transportation System Research Center Transportation System Engineering and ITS Research Group, School of Transportation Engineering, Tongji University. The Beijing Special Control System was established with the great help of J+ traffic Tech Co., Ltd. 


\section{References}

[1] Transportation Research Board (TRB), Proceedings of National Conference on Traffic Incident Management, 2002.

[2] U. S. Department of Transportation (DOT), Fatality Analysis Reporting System, NHTSA, Washington, DC, USA, 2002.

[3] Federal Highway Administration (FHWA), Traffic Signal Preemption for Emergency Vehicles: A Cross-Cutting Study, 2006.

[4] D. Deeter, H. M. Zarean, and D. Register, "Rural ITS Toolbox, Section 3: Emergency Services, Subsection 3. 1 Emergency Vehicle Traffic Signal Preemption," 2001.

[5] D. Bullock, J. Morales, and B. Sanderson, "Evaluation of emergency vehicle signal preemption on the Route 7 Virginia corridor," FHWA-RD-99-70, Federal Highway Administration, Richmond, Va, USA, 1999.

[6] D. Bullock and E. Nelson, "Impact evaluation of emergency vehicle preemption on signalized corridor operation," in Proceedings of the TRB Annual Meeting, Transportation Research Board, Washington, DC, USA, January 2000.

[7] K. Hunter-Zaworski and A. Danaher, "NE Multnomah street Opticom bus signal priority pilot study," Final Report TNW9703, Seattle, Wash, USA, 1995.

[8] Traffic Technologies LLC, "Sonem 2000 digital siren detector," Technical Document for Installation, Traffic Technologies, LLC, 2001.

[9] E. Kwon, K. Sangho, and B. Rober, "Route-based dynamic preemption of traffic signals for emergency vehicles operations," in Transportation Research Record, Transportation Research Board of the National Academies, Washington, DC, USA, 2003.

[10] C. Louisell and J. Collura, "A simple algorithm to estimate emergency vehicle travel time savings on preemption equipped corridors: a method based on a field operational test," in Transportation Research Record, Transportation Research Board of the National Academies, Washington, DC, USA, 2005.

[11] R. Mussa and M. F. Selekwa, "Development of an optimal transition procedure for coordinated traffic signals," Journal of Advances in Transportation Studies, vol. 3, pp. 57-70, 2004.

[12] A. Haghani, H. J. Hu, and Q. Tian, "An optimization model for real-time emergency vehicle dispatching and routing," in Transportation Research Record, Transportation Research Board of the National Academies, Washington, DC, USA, 2003.

[13] I. Yun, B. B. Park, C. K. Lee, and Y. T. Oh, "Comparison of emergency vehicle preemption methods using a hardware-inthe-loop simulation," KSCE Journal of Civil Engineering, vol. 16, no. 6, pp. 1057-1063, 2012.

[14] I. Yun, B. B. Park, C. K. Lee, and Y. T. Oh, "Investigation on the exit phase controls for emergency vehicle preemption," KSCE Journal of Civil Engineering, vol. 15, no. 8, pp. 1419-1426, 2011.

[15] Q. He, K. L. Head, and J. Ding, "Heuristic algorithm for priority traffic signal control," Transportation Research Record, vol. 2259, pp. 1-7, 2011.

[16] P. T. Savolainen, T. K. Datta, I. Ghosh, and T. J. Gates, "Effects of dynamically activated emergency vehicle warning sign on driver behavior at urban intersections," Transportation Research Record, vol. 2149, pp. 77-83, 2010.

[17] T. S. Glickman and E. Erkut, "Assessment of hazardous material risks for rail yard safety," Safety Science, vol. 45, no. 7, pp. 813822, 2007.

[18] M. Davidich and G. Köster, "Towards automatic and robust adjustment of human behavioral parameters in a pedestrian stream model to measured data," Safety Science, vol. 50, no. 5, pp. 1253-1260, 2012.
[19] F. Ozel, "Time pressure and stress as a factor during emergency egress," Safety Science, vol. 38, no. 2, pp. 95-107, 2001.

[20] S. Heliövaara, J.-M. Kuusinen, T. Rinne, T. Korhonen, and H. Ehtamo, "Pedestrian behavior and exit selection in evacuation of a corridor-an experimental study," Safety Science, vol. 50, no. 2, pp. 221-227, 2012.

[21] J.-B. Sheu, "An emergency logistics distribution approach for quick response to urgent relief demand in disasters," Transportation Research E, vol. 43, no. 6, pp. 687-709, 2007.

[22] Y. Deng and F. T. S. Chan, "A new fuzzy dempster MCDM method and its application in supplier selection," Expert Systems with Applications, vol. 38, no. 8, pp. 9854-9861, 2011.

[23] Y. Deng, R. Sadiq, W. Jiang, and S. Tesfamariam, "Risk analysis in a linguistic environment: a fuzzy evidential reasoning-based approach," Expert Systems with Applications, vol. 38, no. 12, pp. 15438-15446, 2011.

[24] A. Hatami-Marbini, M. Tavana, M. Moradi, and F. Kangi, "A fuzzy group electre method for safety and health assessment in hazardous waste recycling facilities," Safety Science, vol. 51, no. 1, pp. 414-426, 2013.

[25] L. Özdamar and C. S. Pedamallu, "A comparison of two mathematical models for earthquake relief logistics," International Journal of Logistics Systems and Management, vol. 10, no. 3, pp. 361-373, 2011.

[26] H. Rakha and Y. Zhang, "Sensitivity analysis of transit signal priority impacts on operation of a signalized intersection," Journal of Transportation Engineering, vol. 130, no. 6, pp. 796804, 2004.

[27] F. Dion, H. Rakha, and Y. Zhang, "Evaluation of potential transit signal priority benefits along a fixed-time signalized arterial," Journal of Transportation Engineering, vol. 130, no. 3, pp. 294303, 2004.

[28] R. J. Baker, J. Collura, J. J. Dale et al., An Overview of Transit Signal Priority-Revised and Updated, ITS America, Washington, DC, USA, 2004.

[29] X. Qin and A. M. Khan, "Control strategies of traffic signal timing transition for emergency vehicle preemption," Transportation Research C, vol. 25, pp. 1-17, 2012.

[30] E. W. Dijkstra, "A note on two problems in connexion with graphs," Numerische Mathematik, vol. 1, pp. 269-271, 1959.

[31] H. Spiess, "Conical volume-delay functions," Transportation Science, vol. 24, no. 2, pp. 153-158, 1990.

[32] U.S. Department of Transportation Federal Highway Administration, Highway History, 2011, http://www.fhwa.dot.gov/highwayhistory/nepa/01.cfm.

[33] T. H. Cormen, C. E. Leiserson, R. L. Rivest, and C. Stein, Introduction to Algorithms, MIT Press, Cambridge, Mass, USA, 2nd edition, 2001. 


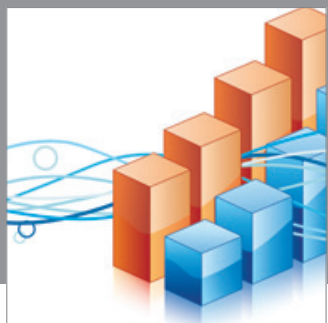

Advances in

Operations Research

mansans

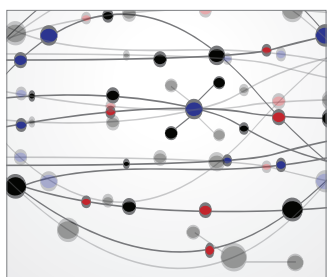

The Scientific World Journal
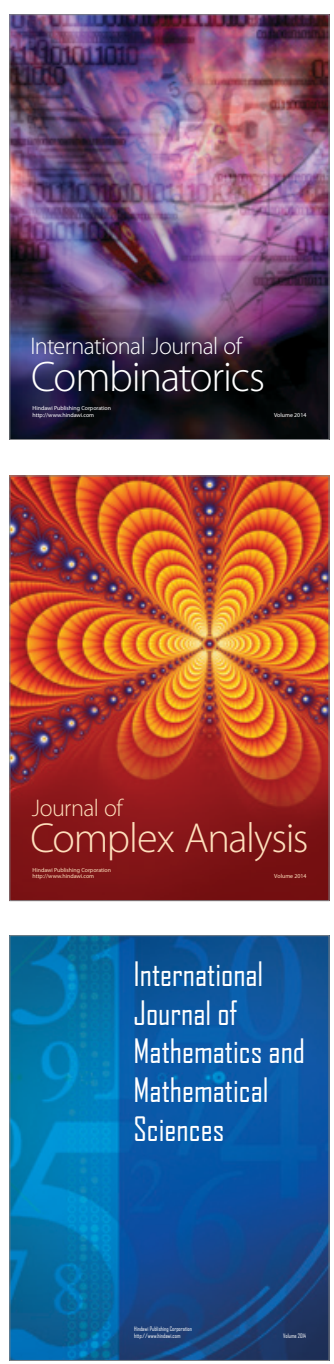
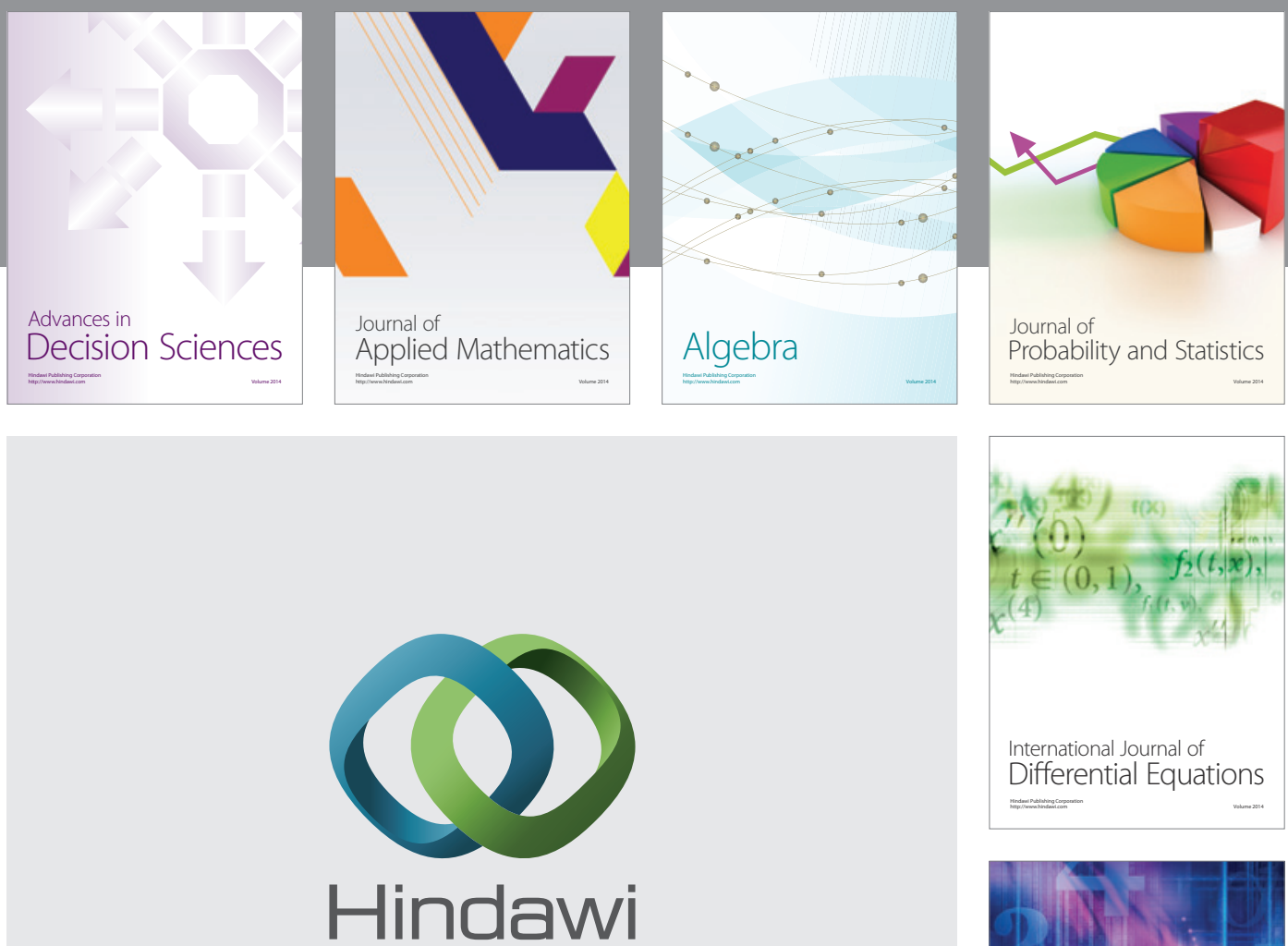

Submit your manuscripts at http://www.hindawi.com
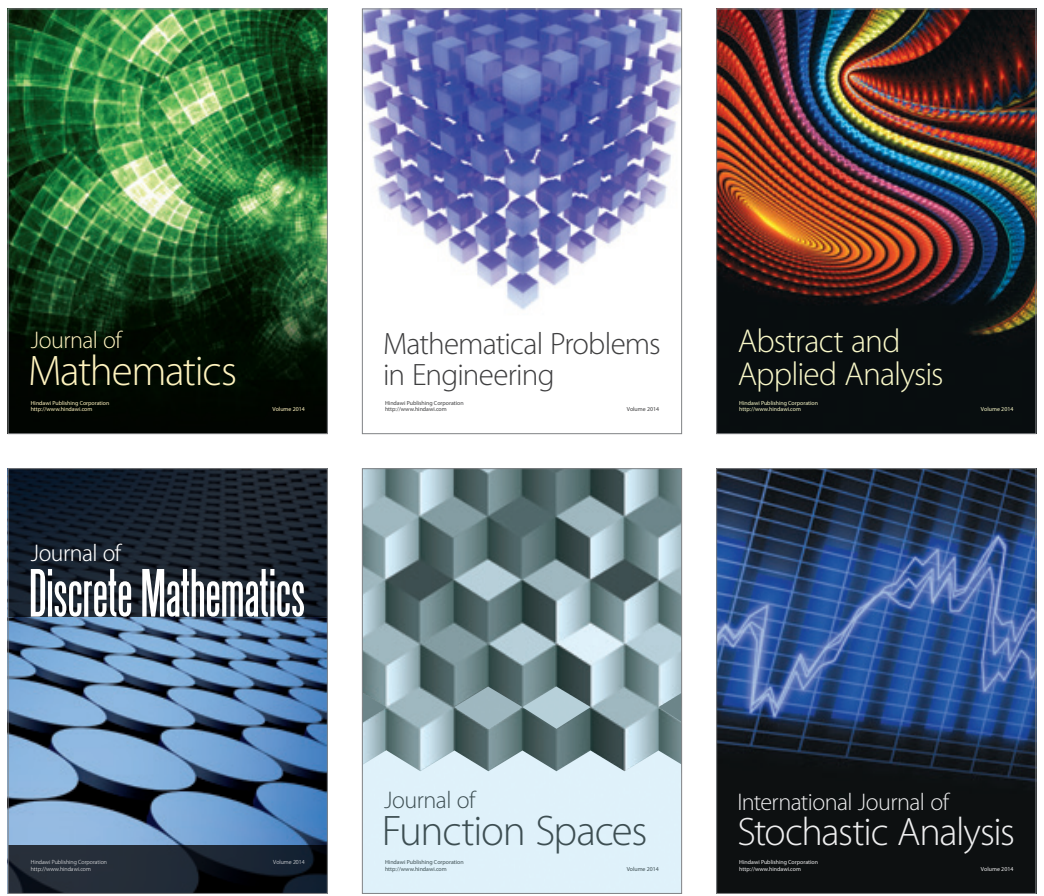

Journal of

Function Spaces

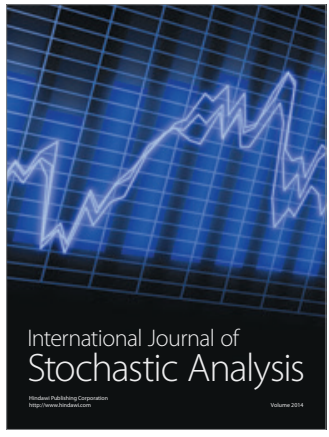

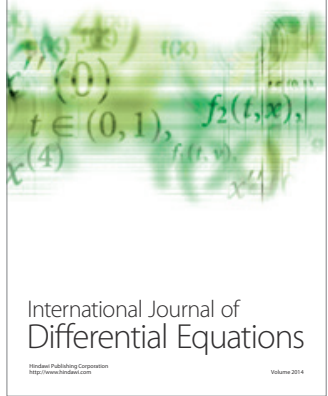
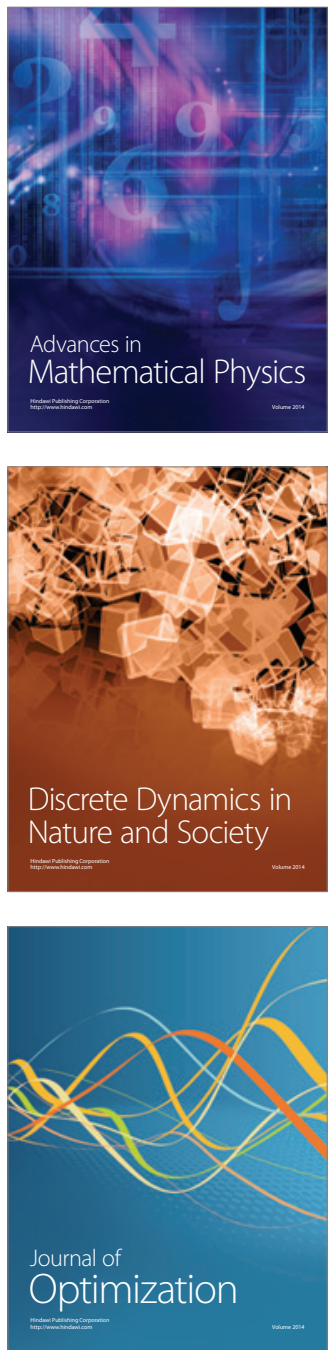